\title{
SOME PROBLEMS CONCERNING KUMMER'S CONGRUENCES FOR THE EULER NUMBERS AND POLYNOMIALS
}

\author{
BY \\ L. CARLITZ AND JACK LEVINE
}

1. Introduction. The Euler polynomial $E_{m}(a)$ of degree $m$ may be defined as the unique polynomial solution of the equation

$$
E_{m}(a+1)+E_{m}(a)=2 a^{m} .
$$

In particular, we have in the notation of Nörlund [3, Chapter 2]

$$
C_{m}=2^{m} E_{m}(0), \quad E_{m}=2^{m} E_{m}(1 / 2) .
$$

Let $p$ be an odd prime, $a$ and $c$ fixed rational numbers that are integral $(\bmod p) ;$ moreover $c \not \equiv 0(\bmod p)$. Put

$$
e_{m}=c^{m} E_{m}(a) \text {. }
$$

Then it is well known that the rational numbers $e_{m}$ satisfy Kummer's congruence [2, Chapter 14]

$$
\sum_{s=0}^{r}(-1)^{s}\left(\begin{array}{l}
r \\
s
\end{array}\right) e_{m+s(p-1)} \equiv 0\left(\bmod \left(p^{m}, p^{r}\right)\right),
$$

where $\left(p^{m}, p^{r}\right)$ denotes the greatest common divisor. Indeed more generally we have

$$
\sum_{s=0}^{r}(-1)^{s}\left(\begin{array}{l}
r \\
s
\end{array}\right) e_{m+s w} \equiv 0\left(\bmod \left(p^{m}, p^{r k}\right)\right),
$$

provided $p^{k-1}(p-1) \mid w$. In particular the congruences (1.4) and (1.5) hold for the numbers $C_{m}, E_{m}$ defined by (1.2).

It is natural to ask whether (1.4) is "best possible" in the following sense. To begin with, for $r=1$, what is the least positive integer $\mu$ such that

$$
e_{m+\mu} \equiv c_{m}(\bmod p)
$$

for all $m \geqq 1$ ? Secondly what is the least positive integer $\mu$ such that

$$
\sum_{s=0}^{r}(-1)^{s}\left(\begin{array}{l}
r \\
s
\end{array}\right) e_{m+s \mu} \equiv 0\left(\bmod \left(p^{m}, p^{r}\right)\right)
$$

Presented to the Society. November 21, 1959; received by the editors August 10, 1959. 
for all $m$ and fixed $r$ ? What is the least positive $t$ such that

$$
\sum_{s=0}^{t}(-1)^{s}\left(\begin{array}{l}
t \\
s
\end{array}\right) e_{m+s(p-1)} \equiv 0\left(\bmod \left(p^{m}, p^{r}\right)\right)
$$

for all $m$ and fixed $r$ ? Similarly for (1.5) we seek (i) the least positive $\mu$ such that

$$
e_{m+\mu} \equiv e_{m}\left(\bmod \left(p^{m}, p^{k}\right)\right)
$$

for all $m$ and fixed $k$; (ii) the least positive $\mu$ such that

$$
\sum_{s=0}^{r}(-1)^{s}\left(\begin{array}{l}
r \\
s
\end{array}\right) e_{m+s \mu} \equiv 0\left(\bmod \left(p^{m}, p^{r k}\right)\right)
$$

for all $m, r$ and fixed $k$; (iii) the least positive $t$ such that

$$
\sum_{s=0}^{t}(-1)^{s}\left(\begin{array}{l}
t \\
s
\end{array}\right) e_{m+s w} \equiv 0\left(\bmod \left(p^{m}, p^{r k}\right)\right)
$$

for all $m$ and fixed $r, k$, where $w=p^{k-1}(p-1)$.

We shall show that for (1.6) and (1.7) $\mu=p-1$; for (1.8) we show that $t=r$ provided $2^{p} \not \equiv 2\left(\bmod p^{2}\right)$ and $p \geqq 2 r^{2}+1$. For (1.9) and (1.10) we show that $\mu=p^{k-1}(p-1)$ provided $p>3$; for (1.11) we show that $t=r$ provided $2^{p} \not \equiv 2\left(\bmod p^{2}\right)$ and $p \geqq 2 r^{2}+1$. It is then clear what is meant by saying that (1.4) is best possible; similarly for (1.5) with $w=p^{k-1}(p-1)$. While (1.6), (1.7), (1.8) are special cases of (1.9), (1.10), (1.11), respectively, it is convenient to treat separately the indicated cases.

We observe that in certain of the results enumerated above, the case of small $p$ is left open. A special discussion of (4.3) below when $p=3$ suggests that some of the general results may indeed require modification for small values of $p$.

It should be observed that if $m$ in (1.4) or (1.5) is restricted to an arithmetic progression, then the theorems of this paper no longer apply. For a special result of this nature see Theorem 9 below.

2. In order to show that $\mu$ in (1.6) is equal to $p-1$ we require the following preliminary result. Put

$$
D_{p}=\left|e_{i+j+1}\right| \quad(i, j=0,1, \cdots, p-2),
$$

a determinant of order $p-1$. We shall show that

$$
D_{p} \equiv \neq 1(\bmod p) \text {. }
$$

Making use of the fact that

$$
e_{m+p-1} \equiv e_{m}(\bmod p)
$$

for $m \geqq 1$, it is easily verified that $D_{p} \equiv \neq C_{p}(\bmod p)$, where $C_{p}$ is the following circulant of order $p-1$ : 


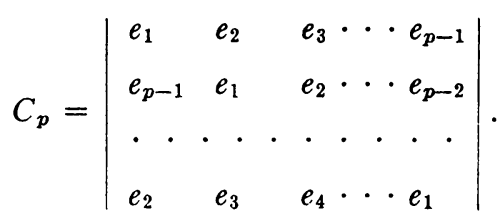

Now the familiar formula for the factorization of a circulant suggests the following analog:

$$
C_{p} \equiv \prod_{r=1}^{p-1} \sum_{s=1}^{p-1} r^{s-1} e_{s}(\bmod p)
$$

Indeed, if $\left(a_{r-8}\right)(r, s=1, \cdots, p-1)$ is an arbitrary circulant matrix of order $p-1$, then

$$
\left(r^{s-1}\right)\left(a_{r-s-1}\right)\left(s^{1-r}\right)=\left(b_{r s}\right)
$$

where

$$
b_{r s}=\sum_{j=1}^{p-1} \sum_{k=1}^{p-1} r^{j-1} a_{j-k} s^{1-k} \equiv \sum_{i=0}^{p-2} \sum_{k=1}^{p-1} r^{i+k-1} a_{i} s^{1-k} \equiv \sum_{i=0}^{p-2} r^{i} a_{i} \sum_{k=0}^{p-2}\left(r s^{-1}\right)^{k}(\bmod p) .
$$

Since the inner sum $\equiv 0(\bmod p)$ unless $r \equiv s(\bmod p)$, we get

$$
b_{r s} \equiv-\delta_{r s} \sum_{i=0}^{p-2} r^{i} a_{i}
$$

Also

$$
\left(r^{2-1}\right)\left(s^{1-r}\right)=\left(\sum_{j=1}^{p-1} r^{j-1} s^{1-j}\right) \equiv\left(-\delta_{r \varepsilon}\right)
$$

Hence (2.3) follows at once.

In the next place, since

$$
E_{n}(a+x)=\sum_{s=0}^{n}\left(\begin{array}{l}
n \\
s
\end{array}\right) x^{n-s} E_{s}(a)
$$

we have

$$
E_{p-1}(a-x) \equiv \sum_{s=0}^{p-1} x^{p-1-s} E_{s}(a)(\bmod p)
$$

Replacing $x$ by $r^{-1}$, this becomes

$$
E_{p-1}\left(a-\frac{1}{c r}\right) \equiv 1+r \sum_{s=1}^{p-1} r^{s-1} c^{8} E_{s}(a) \equiv 1+r \sum_{s=1}^{p-1} r^{s-1} e_{s}(\bmod p) .
$$

Now it follows from (1.1) that 


$$
\sum_{s=1}^{n}(-1)^{s} s^{k}=\frac{1}{2}(-1)^{n} E_{k}(n+1)+\frac{1}{2} E_{k}(0) .
$$

For $k=p-1,(2.5)$ becomes (since $E_{p-1}(0)=0$ )

$$
\sum_{s=1}^{n}(-1)^{s} \equiv \frac{1}{2}(-1)^{n} E_{p-1}(n+1)(\bmod p) \quad(0 \leqq n<p) .
$$

Since

$$
\sum_{s=1}^{n}(-1)^{\bullet}=\left\{\begin{aligned}
-1 & (n \text { odd }) \\
0 & (n \text { even })
\end{aligned}\right.
$$

we get

$$
E_{p-1}(m) \equiv\left\{\begin{array}{ll}
2 & (m \text { even }), \\
0 & (m \text { odd }),
\end{array} \quad(1 \leqq m \leqq p) .\right.
$$

Returning to (2.4), we define $m$ by means of

$$
m \equiv a-\frac{1}{c r}(\bmod p) \quad(1 \leqq m \leqq p) .
$$

Then, using (2.6), it is clear that

$$
r \sum_{s=1}^{p-1} r^{s-1} e_{s} \equiv \neq 1(\bmod p) \quad(1 \leqq r \leqq p-1) .
$$

This yields

$$
\prod_{r=1}^{p-1} \sum_{s=1}^{p-1} r^{s-1} e_{s} \equiv \neq 1(\bmod p)
$$

Substituting in (2.3), we get (2.2).

3 . We now prove

TheOREM 1. Assume that the numbers $e_{m}$ defined by (1.3) satisfy the congruence

$$
e_{m+k}+c_{1} e_{m+k-1}+\cdots+c_{k} e_{m} \equiv 0(\bmod p),
$$

for all $m \geqq 1$, where $c_{1}, \cdots, c_{k}$ are integral $(\bmod p)$ and independent of $m$. Then $k \geqq p-1$.

If we assume $k<p-1$, it follows from $(3.1)$ that $D_{p} \equiv 0(\bmod p)$. Since this contradicts (2.2), the theorem is proved.

As an immediate corollary of Theorem 1 we have

Theorem 2. The least positive integer $\mu$ such that

$$
e_{m+\mu} \equiv e_{m}(\bmod p)
$$


for all $m \geqq 1$, is $\mu=p-1$.

Turning next to the congruence

$$
\sum_{s=0}^{r}(-1)^{s}\left(\begin{array}{c}
r \\
s
\end{array}\right) e_{m+s \mu} \equiv 0\left(\bmod \left(p^{m}, p^{r}\right)\right)
$$

for all $m$ and fixed $r$, we assume that $\mu$ is the smallest positive integer for which (3.2) holds. It follows that

$$
\sum_{s=0}^{r}(-1)^{s}\left(\begin{array}{l}
r \\
s
\end{array}\right) e_{m+s \mu} \equiv 0(\bmod p)
$$

for all $m \geqq 1$.

The polynomials $\left(1-x^{\mu}\right)^{r}$ and $1-x^{p-1}$ have a greatest common divisor $(\bmod p)$ of the form $1-x^{d}$, where $d \mid p-1$. Thus there exist polynomials $A(x), B(x)$ with integral coefficients such that

$$
\left(1-x^{\mu}\right)^{r} A(x)+\left(1-x^{p-1}\right) B(x) \equiv 1-x^{d}(\bmod p) .
$$

Consequently we get (symbolically)

$$
e^{m}\left(1-e^{\mu}\right)^{r} A(e)+e^{m}\left(1-e^{p-1}\right) B(e) \equiv e^{m}\left(1-e^{d}\right)(\bmod p) .
$$

Using (3.3) and (1.4) this reduces to

$$
e_{m+d} \equiv e_{m}(\bmod p)
$$

for all $m \geqq 1$. In view of Theorem $2, d=p-1$. Then

$$
\left(1-x^{p-1}\right) \mid\left(1-x^{\mu}\right)^{r}(\bmod p)
$$

which implies $\mu=p-1$. This completes the proof of

Theorem 3. The least positive integer $\mu$ such that

$$
\sum_{s=0}^{r}(-1)^{s}\left(\begin{array}{l}
r \\
s
\end{array}\right) e_{m+s \mu} \equiv 0\left(\bmod \left(p^{m}, p^{r}\right)\right)
$$

for all $m$ and fixed $r$ is $\mu=p-1$.

4. We consider next the congruence

$$
\sum_{s=0}^{t}(-1)^{s}\left(\begin{array}{l}
t \\
s
\end{array}\right) e_{m+s(p-1)} \equiv 0\left(\bmod \left(p^{m}, p^{r}\right)\right)
$$

and seek the least positive integer $t$ such that (4.1) holds for all $m$ and fixed $r$. Clearly $1 \leqq t \leqq r$; also if (4.1) holds for a certain $t$ it will hold for all larger $t$. Hence it will suffice to show that

$$
\sum_{s=0}^{t}(-1)^{s}\left(\begin{array}{l}
t \\
s
\end{array}\right) e_{m+s(p-1)} \equiv 0\left(\bmod \left(p^{m}, p^{t+1}\right)\right)
$$


for all $m$ and any fixed $t \geqq 1$ is impossible.

We first examine the case $t=1$, namely the congruence

$$
c_{m+p-1} \equiv e_{m}\left(\bmod p^{2}\right) \quad(m \geqq 2) .
$$

Since, for all $m, E_{m}(a+p) \equiv E_{m}(a)+m p E_{m-1}(a)\left(\bmod p^{2}\right)$, it follows from (1.3) and (4.3) that

$$
c^{p-1} E_{m+p-1}(a+p)-E_{m}(a+p) \equiv-p E_{m-1}(a)\left(\bmod p^{2}\right)
$$

for $m \geqq 2$. Also (compare (2.5))

$$
E_{k}(a+p)+E_{k}(a)=2 \sum_{s=0}^{p-1}(-1)^{s}(a+s)^{k}
$$

so that

$$
\begin{aligned}
\left\{c^{p-1} E_{m+p-1}(a-p)-E_{m}(a+p)\right\} & +\left\{c^{p-1} E_{m+p-1}(a)-E_{m}(a)\right\} \\
& =2 \sum_{s=0}^{p-1}(-1)^{s}(a+s)^{m}\left\{(a+s)^{p-1} c^{p-1}-1\right\} .
\end{aligned}
$$

Then, using (4.3) and (4.4), this reduces to

$$
2 \sum_{s=0}^{p-1}(-1)^{s}(a+s)^{m}\left\{(a+s)^{p-1} c^{p-1}-1\right\} \equiv-p E_{m-1}(a)\left(\bmod p^{2}\right)
$$

for all $m \geqq 2$. It is convenient to replace $m$ by $m+1$, so that (4.5) becomes

$$
\text { (4.6) } 2 \sum_{s=n}^{p-1}(-1)^{s}(a+s)^{m-1}\left\{(a+s)^{p-1} c^{p-1}-1\right\} \equiv-p E_{m}(a)\left(\bmod p^{2}\right)
$$

valid for all $m \geqq 1$. We now multiply both sides of $(4.6)$ by $(a+j)^{p-1-m}$, where $1 \leqq m \leqq p-1$ and $0 \leqq j \leqq p-1$; the value $j=j_{0}$ such that $p \mid a+j$ is omitted. Since

$$
\sum_{m=1}^{p-1}(a-j)^{p-1-m}(a+s)^{m} \equiv\left\{\begin{array}{rll}
-1 & (\bmod p) & (j=s) \\
0 & (\bmod p) & (j \neq s)
\end{array}\right.
$$

we get

$$
\text { (4.7) } 2(-1)^{i}(a+j)\left\{(a+j)^{p-1} c^{p-1}-1\right\} \equiv p \sum_{m=1}^{p-1}(a+j)^{p-1-m} E_{m}(a)\left(\bmod p^{2}\right)
$$

for all $j \neq j_{0}$ such that $0 \leqq j \leqq p-1$.

In the next place, since

$$
E_{p-1}(a-x) \equiv \sum_{m=0}^{p-1} x^{p-1-m} E_{m}(a)(\bmod p)
$$

it is clear that 


$$
\begin{aligned}
\sum_{m=1}^{p-1}(a+j)^{p-1-m} E_{m}(a) & \equiv E_{p-1}(-j)-(a+j)^{p-1} \equiv E_{p-1}(p-j)-1 \\
& \equiv(-1)^{j-1}(\bmod p),
\end{aligned}
$$

where at the last step we have used (2.6). Consequently (4.7) becomes

$$
(a+j)^{p} c^{p}-(a+j) c \equiv-\frac{1}{2} p c\left(\bmod p^{2}\right)
$$

for all $j \neq j_{0}, 0 \leqq j \leqq p-1$.

If we sum (4.8) over $j$ we get

$$
\begin{aligned}
\sum_{j=0}^{p-1}(a+j)^{p} c^{p}-\left(a+j_{0}\right)^{p} c^{p}-\sum_{j=0}^{p-1}(a+j) c & +\left(a+j_{0}\right) c \\
& \equiv-\frac{1}{2}(p-1) c\left(\bmod p^{2}\right) .
\end{aligned}
$$

If $B_{n}(x)$ is the Bernoulli polynomial of degree $n$, we have

$$
\begin{aligned}
\sum_{j=0}^{p-1}(a+j)^{p} & =\frac{B_{p+1}(a+p)-B_{p+1}(a)}{p+1} \equiv p B_{p}(a)\left(\bmod p^{2}\right), \\
B_{p}(a) & =\sum_{s=0}^{p}\left(\begin{array}{c}
p \\
s
\end{array}\right) B_{s} a^{p-s}=a^{p}+\sum_{s=1}^{p-2}\left(\begin{array}{c}
p \\
s
\end{array}\right) B_{s} a^{p-s}+p B_{p-1} a+B_{p} \\
& \equiv a^{p}-a(\bmod p),
\end{aligned}
$$

since by the Staudt-Clausen theorem

$$
p B_{p-1} \equiv-1(\bmod p) .
$$

Thus

$$
\sum_{j=0}^{p-1}(a+j)^{p} \equiv 0\left(\bmod p^{2}\right)
$$

and (4.9) reduces to

$$
-p a c-\frac{1}{2} p(p-1) c+\left(a+j_{0}\right) c \equiv-\frac{1}{2} p(p-1) c\left(\bmod p^{2}\right),
$$

which simplifies to

$$
a+j_{0} \equiv p a\left(\bmod p^{2}\right) .
$$

If $j_{0} \neq 0$ or $p-1$, allowable values for $j$ in (4.8) are $j_{0} \neq 1$. For $j=j_{0}+1$, (4.8) becomes

$$
c^{p}-c \equiv\left(a-\frac{1}{2}\right) p c\left(\bmod p^{2}\right),
$$


while for $j=j_{0}-1$ we get

$$
c^{p}-c \equiv-\left(a-\frac{1}{2}\right) p c\left(\bmod p^{2}\right) .
$$

Consequently $a \equiv(\bmod p) / 2 ;$ by $(4.10)$ this implies

$$
a \equiv \frac{1}{2}\left(\bmod p^{2}\right) \quad\left(j_{0} \neq 0, p-1\right) .
$$

Also (4.10) yields

$$
\begin{aligned}
& a \equiv 0\left(\bmod p^{2}\right) \\
& a \equiv 1\left(\bmod p^{2}\right)
\end{aligned}
$$$$
\left(j_{0}=0\right),
$$$$
\left(j_{0}=p-1\right) \text {. }
$$

When (4.11) holds we have

$$
c^{p} \equiv c\left(\bmod p^{2}\right),
$$

so that $(4.8)$ reduces to

$$
\left(\frac{1}{2}+j\right)^{p}-\left(\frac{1}{2}+j\right) \equiv-\frac{1}{2} p\left(\bmod p^{2}\right) .
$$

Put $j=(p-1) / 2+k$, so that $(4.14)$ becomes

$$
k^{p}-k \equiv 0\left(\bmod p^{2}\right) \quad\left(k=1, \cdots, \frac{1}{2}(p-1)\right) .
$$

Now Brauer [1] has proved that for $p \geqq 5$, the smallest primitive root $g\left(\bmod p^{2}\right)$ is less than $p$. If this least primitive root $<p / 2$, then $(4.15)$ is clearly impossible; if $g>p / 2$, then $p-g<p / 2$ and belongs to an exponent $\left(\bmod p^{2}\right)$ that is a multiple of $p$, so that again (4.15) is contradicted.

In the next place, when (4.12) holds, (4.8) becomes

$$
{ }^{i p} c^{p}-j c \equiv-\frac{1}{2} p c\left(\bmod p^{2}\right) .
$$

Let

$$
j^{p} \equiv j+p m_{j}\left(\bmod p^{2}\right) \quad(j=1, \cdots, p-1) .
$$

Since (4.16) with $j=1$ gives

$$
c^{p} \equiv c-\frac{1}{2} p c\left(\bmod p^{2}\right),
$$

we have

$$
j^{p} c^{p} \equiv j c+p c m_{j}-\frac{1}{2} p c j\left(\bmod p^{2}\right) .
$$


Comparing this with (4.16) we get

$$
m_{j} \equiv \frac{1}{2}(j-1)(\bmod p)
$$

so that

$$
j^{p} \equiv j+\frac{1}{2} p(j-1)\left(\bmod p^{2}\right) \quad(j=1, \cdots, p-1) .
$$

It is easy to see that (4.18) is impossible for $p \geqq 5$. Indeed for $j=2$ we have

$$
2^{p} \equiv 2+\frac{1}{2} p\left(\bmod p^{2}\right)
$$

and therefore

$$
4^{p} \equiv 4+2 p\left(\bmod p^{2}\right) .
$$

On the other hand, for $j=4,(4.18)$ is

$$
4^{p} \equiv 4+\frac{3}{2} p\left(\bmod p^{2}\right)
$$

In the third place since $E_{m}(1)=-E_{m}(0)$ for $m \geqq 1$, it is clear that (4.13) and (4.12) are equivalent cases. We have therefore proved the impossibility of (4.3) for all $p \geqq 5$. We may state

THEOREM $4(p>3)$. The congruence

$$
e_{m+p-1} \equiv e_{m}\left(\bmod p^{2}\right)
$$

cannot hold for all $m \geqq 2$.

That the condition $p>3$ is necessary (for some values of $a$ and $c$ ) can be seen as follows. Since

$$
e_{m+4}-2 e_{m+2}+e_{m} \equiv 0(\bmod 9)
$$

for all $m \geqq 2$, the assumption that

$$
e_{m+2} \equiv e_{m}(\bmod 9)
$$

for some fixed $m=m_{0} \geqq 2$ implies that (4.20) holds for all $m \geqq m_{0}$, $m \equiv m_{0}(\bmod 2)$.

In particular, since $E_{2}=-1, E_{4}=5$,

$$
E_{4}\left(\frac{1}{2}\right) \equiv E_{2}\left(\frac{1}{2}\right)(\bmod 9)
$$

and therefore 


$$
E_{m+2}\left(\frac{1}{2}\right) \equiv E_{m}\left(\frac{1}{2}\right)(\bmod 9)
$$

for all $m \geqq 2$ (incidentally including odd values). Also $C_{1}=-1, C_{3}=2$, $C_{5}=-16$, we have

$$
C_{m+2} \equiv C_{m}(\bmod 9)
$$

$$
(m \geqq 2) \text {. }
$$

5. We now return to (4.2). Since

$$
E_{m}\left(a+p^{t}\right) \equiv E_{m}(a)+m p^{t} E_{m-1}(a)\left(\bmod p^{t+1}\right) \quad(t \geqq 1),
$$

it follows that

$$
\begin{aligned}
\sum_{s=0}^{t}(-1)^{s}\left(\begin{array}{l}
t \\
s
\end{array}\right) c^{m+s(p-1)} E_{m+s(p-1)}\left(a+p^{t}\right) & \\
& \equiv \sum_{s=0}^{t}(-1)^{s}\left(\begin{array}{l}
t \\
s
\end{array}\right) e_{m+s(p-1)}\left(\bmod p^{t+1}\right),
\end{aligned}
$$

since

$$
\begin{aligned}
\sum_{s=0}^{t}(-1)^{\cdot}\left(\begin{array}{l}
t \\
s
\end{array}\right)(m+s(p-1)) c^{m+s(p-1)} E_{m+\mho(p-1)-1}(a) \\
\equiv \sum_{s=0}^{t}(-1)^{s}\left(\begin{array}{l}
t \\
s
\end{array}\right)(m+s(p-1)) E_{m-1}(a) \equiv 0(\bmod p)
\end{aligned}
$$

for $t \geqq 2$ and $m \geqq 2$. Hence assuming that (4.2) holds we get

$$
\sum_{s=0}^{t}(-1) \cdot\left(\begin{array}{l}
t \\
s
\end{array}\right) c^{m+s(p-1)} E_{m+s(p-1)}\left(a+p^{t}\right) \equiv 0\left(\bmod p^{t+1}\right)
$$

for $t \geqq 2, m \geqq t+1$. Since we have also

$$
\sum_{j=0}^{p l-1}(-1)^{i}(a+j)^{m}=\frac{1}{2} E_{m}\left(a+p^{t}\right)+\frac{1}{2} E_{m}(a),
$$

(5.1) yields

$$
\sum_{j=0}^{p t-1}(-1)^{j}(a+j)^{m} c^{m}\left\{(a+j)^{p-1} c^{p-1}-1\right\}^{t} \equiv 0\left(\bmod p^{t+1}\right) .
$$

It will be convenient to rewrite this as

$$
\sum_{s=0}^{p t-1}(-1)^{s}(a+s)^{m}\left\{(a+s)^{p} c^{p}-(a+s) c\right\}^{t} \equiv 0\left(\bmod p^{t+1}\right),
$$

which is asserted for $t \geqq 2$ and $m \geqq 1$. Now in the left member of (5.2) replace $s$ by $j+p k$, where $0 \leqq j \leqq p-1,0 \leqq k \leqq p^{t-1}-1$. Then (5.2) becomes 


$$
\begin{aligned}
\sum_{j=0}^{p-1}(-1)^{j} \sum_{k=0}^{p t-1-1}(-1)^{k}(a+j+p k)^{m}\left\{(a+j+p k)^{p} c^{p}\right. & -(a+j+p k) c\}^{t} \\
& \equiv 0\left(\bmod p^{t+1}\right)
\end{aligned}
$$

Since

$$
(a+j+p k)^{p} \equiv(a+j)^{p}\left(\bmod p^{2}\right),
$$

the inner sum in (5.3)

$$
\begin{aligned}
& \equiv(a+j)^{m} \sum_{k=0}^{p t-1-1}(-1)^{k}\left\{(a+j)^{p} c^{p}-(a+j) c-p k\right\}^{\imath} \\
& \equiv(a+j)^{m} p^{t} \sum_{k=0}^{p t-1-1}(-1)^{k}\left(A_{j}-k\right)^{t} \\
& \equiv(a+j)^{m} p^{t} \sum_{k=0}^{t-1-1}(-1)^{k}\left(A_{j}-p^{t-1}+1+k\right)^{t} \\
& \equiv \frac{1}{2}(a+j)^{m} p^{t}\left\{E_{t}\left(A_{j}+1\right)+E_{t}\left(A_{j}+1-p^{t-1}\right)\right\}\left(\bmod p^{t+1}\right)
\end{aligned}
$$

where

$$
A_{j}=\left\{(a+j)^{p} c^{p}-(a+j) c\right\} / p .
$$

Since $t \geqq 2$, it is clear that

$$
E_{t}\left(A_{j}+1-p^{t-1}\right) \equiv E_{t}\left(A_{j}+1\right)(\bmod p) .
$$

Consequently (5.3) reduces to

$$
\sum_{j=0}^{p-1}(-1)^{j}(a+j)^{m} E_{t}\left(A_{j}+1\right) \equiv 0(\bmod p)
$$

with $A_{j}$ defined by (5.4). It then follows from (5.5) (compare the proof of (4.8)) that

$$
E_{t}\left(A_{j}+1\right) \equiv 0(\bmod p)
$$

for all $j \neq j_{0}, 0 \leqq j<p$, where $a+j_{0} \equiv 0(\bmod p)$.

It is clear from (5.6) that $A_{j}$ can take on at most $t$ distinct values $(\bmod p)$. We shall show that for $t$ fixed and $p$ sufficiently large this is impossibleprovided

$$
2^{p} \not \equiv 2\left(\bmod p^{2}\right)
$$

Put

$$
N=\left[(p / 2)^{1 / 2}\right],
$$

where $[x]$ is the greatest integer $\leqq x$. Then, if (5.7) holds, at least $N$ of the numbers 


$$
(a+j)^{p} c^{p}-(a+j) c
$$$$
\left(0 \leqq j<p, j \neq j_{0}\right)
$$

are distinct $\left(\bmod p^{2}\right)$. If we put $j=j_{0}+j^{\prime}$ this is equivalent to the assertion that at least $N$ of the numbers

$$
i^{\prime p} c^{p}=j^{\prime} c \quad\left(-j_{0} \leqq j^{\prime}<p-j_{0}, j^{\prime} \neq 0\right)
$$

are distinct $\left(\bmod p^{2}\right)$. If at most $N-1$ of the numbers $(5.10)$ are distinct $\left(\bmod p^{2}\right)$, then since $p-1 \geqq 2 N^{2}$ it follows that, there exist at least $2 N$ distinct numbers $j_{s}^{\prime}(\bmod p)$ such that

$$
j_{s}^{\prime} c^{p}-j_{s}^{\prime} c \equiv k p\left(\bmod p^{2}\right) \quad(s=1, \cdots, 2 N)
$$

for some fixed $k$. Replacing $j_{s}^{\prime}$ by $p \neq j_{s}^{\prime}$ we get a congruence of the same form with possibly a different $k$. Hence we may assume that (5.11) is satisfied by $N$ values of $j_{s}^{\prime}$ such that $1 \leqq j_{s}^{\prime}<p / 2$. If we put

$$
2^{p} \equiv 2+w p\left(\bmod p^{2}\right)
$$$$
(p \neq w)
$$

we get

$$
\left(2 j_{s}^{\prime}\right)^{p} c^{p}-2 j_{s}^{\prime} c \equiv\left(2 k+j_{s}^{\prime} c w\right) p\left(\bmod p^{2}\right) \quad(s=1, \cdots, N) .
$$

These $N$ numbers are distinct $\left(\bmod p^{2}\right)$ so that we have a contradiction. Hence we have proved the assertion about (5.9). For the proof compare Vandiver [4].

It therefore follows that (5.6) is impossible when $N>t$, or, what is the same thing, when

$$
p \geqq 2(t+1)^{2}+1 .
$$

We have now proved the impossibility of the congruence (4.2) subject to the condition (5.7) and (5.12). We may accordingly state the following

THEOREM 5. The least positive integer $t$ such that

$$
\sum_{s=0}^{t}(-1)^{s}\left(\begin{array}{l}
t \\
s
\end{array}\right) e_{m+s(p-1)} \equiv 0\left(\bmod \left(p^{m}, p^{r}\right)\right),
$$

for all $m$ and fixed $r \geqq 2$ is given by $t=r$ provided $2^{p} \not \equiv 2\left(\bmod p^{2}\right)$ and

$$
p \geqq 2 r^{2}+1 \text {. }
$$

6. The symbolic identity

$$
e^{m}\left(e^{p(p-1)}-1\right)=\sum_{r=1}^{p}\left(\begin{array}{l}
p \\
r
\end{array}\right) e^{m}\left(e^{p-1}-1\right)^{r},
$$

where after expansion $e^{n}$ is replaced by $e_{n}$, evidently implies (for $m \geqq 3$ )

$$
e^{m}\left(e^{p(p-1)}-1\right) \equiv p e^{m}\left(e^{p-1}-1\right)\left(\bmod p^{3}\right) .
$$

Thus the impossibility of (4.3) for $p>3$ implies that 


$$
e_{m+p(p-1)}-e_{m} \equiv 0\left(\bmod p^{3}\right)
$$

for all $m \geqq 3$ is impossible for $p>3$.

Much more can be obtained in this way. In the identity

$$
x^{p}-1=\sum_{s=1}^{p}\left(\begin{array}{l}
p \\
s
\end{array}\right)(x-1)^{s}
$$

replace $x$ by $e^{p^{k-1}(p-1)}$, so that

$$
e^{p^{k}(p-1)}-1=\sum_{s=1}^{p}\left(\begin{array}{l}
p \\
s
\end{array}\right)\left(e^{p^{k-1}(p-1)}-1\right)^{s} .
$$

This yields

$$
e^{m}\left(e^{p^{k}(p-1)}-1\right) \equiv p e^{m}\left(e^{p^{k-1}(p-1)}-1\right)\left(\bmod p^{k+2}\right)
$$

for all $m \geqq k+2$. The case $k=1$ is that just discussed. For $k=2$ we have

$$
e^{m}\left(e^{p^{2}(p-1)}-1\right) \equiv p e^{m}\left(e^{p(p-1)}-1\right)\left(\bmod p^{4}\right) .
$$

We infer that

$$
e^{m}\left(e^{p^{2}(p-1)}-1\right) \equiv 0\left(\bmod p^{4}\right)
$$

for all $m \geqq 4$ is impossible $(p>3)$. Generally, by means of (6.4), an easy induction shows that

$$
e^{m}\left(e^{p^{k-1}(p-1)}-1\right) \equiv 0\left(\bmod p^{k+1}\right)
$$

for all $m \geqq k+1$ is impossible when $p>3$.

In the congruence

$$
e_{m+\mu} \equiv e_{m}\left(\bmod \left(p^{m}, p^{k}\right)\right),
$$

it is clear from Theorem 2 that $p-1 \mid \mu$. Therefore, applying the result obtained for (6.5), we may state

THEOREM $6(p>3)$. The least positive integer $\mu$ such that (6.6) holds for all $m$ and fixed $k \geqq 1$ is given by

$$
\mu=p^{k-1}(p-1) .
$$

Turning next to the congruence

$$
\sum_{s=0}^{r}(-1)^{\cdot}\left(\begin{array}{l}
r \\
s
\end{array}\right) e_{m+s \mu} \equiv 0\left(\bmod \left(p^{m}, p^{r k}\right)\right),
$$

which is assumed to hold for all $m$ and fixed $r, k$, it is first of all easy to see that $p-1 \mid \mu$. Indeed, for $m \geqq 1$, (6.8) obviously implies

$$
e^{m}\left(e^{\mu^{\prime}}-1\right)^{r} \equiv 0\left(\bmod p^{r}\right)
$$


with minimum $\mu^{\prime}=p-1$; since

$$
\left(x^{p-1}-1\right)^{r} \mid\left(x^{\mu}-1\right)^{r}(\bmod p)
$$

if and only if $p-1 \mid \mu$, the assertion above follows at once.

If we next raise both sides of (6.3) to the $r$ th power we get an identity of the following kind:

$$
\begin{aligned}
\left(x^{p}-1\right)^{r} & =\left\{p(x-1) f(x-1)+(x-1)^{p}\right\}^{r} \\
& =p^{r}(x-1)^{r} f^{r}(x-1)+\cdots+(x-1)^{p r} .
\end{aligned}
$$

where $f(x-1)$ is a polynomial with integral coefficients. Now replacing $x$ by $e^{p^{k-1}(p-1)}-1$, we get

$$
e^{m}\left(e^{p^{k}(p-1)}-1\right)^{r} \equiv p^{r} e^{m}\left(e^{p^{k-1}(p-1)}-1\right)^{r}\left(\bmod p^{k r+r+1}\right)
$$

for all $m \geqq k r+r+1$. An induction with respect to $k$ yields the following

THEOREM $7(p>3)$. The least positive integer $\mu$ such that (6.8) holds for all $m$ and fixed $k \geqq 1, r \geqq 1$ is given by (6.7).

Finally we consider the congruence

$$
\sum_{s=0}^{t}(-1)^{s}\left(\begin{array}{l}
t \\
s
\end{array}\right) e_{m+s w} \equiv 0\left(\bmod \left(p^{m}, p^{r k}\right)\right)
$$

where $w=p^{k-1}(p-1)$, for fixed $r, k$. Clearly we have $t \leqq r$. It will suffice to show that (6.10) is impossible when $t=r-1$. For, assuming that (6.10) holds when $t=r-1$, we get by repeated application of (6.9)

$$
\begin{aligned}
e^{m}\left(e^{p^{k-1}(p-1)}-1\right)^{r-1} \equiv p^{r-1} e^{m}\left(e^{p^{k-2}(p-1)}-1\right)^{r-1} & \\
& \equiv \cdots \equiv p^{(k-1)(r-1)} e^{m}\left(e^{p-1}-1\right)^{r-1}\left(\bmod p^{k(r-1)+1}\right)
\end{aligned}
$$

for $m \geqq k t-k$. Since by Theorem 4

$$
e^{m}\left(e^{p-1}-1\right)^{r-1} \not \equiv 0\left(\bmod p^{r}\right)
$$

when certain conditions are satisfied, it follows that

$$
e^{m}\left(e^{p^{k-1}(p-1)}-1\right)^{r-1} \not \equiv 0\left(\bmod p^{k(r-1)+1}\right)
$$

for all $m \geqq k(r-1)+1$. This completes the proof of

THEOREM 8. The least positive integer $t$ for which the congruence (6.10) is satisfied for all $m$ and fixed $r \geqq 1, k \geqq 1$, is given by $t=r$, provided $2^{p} \not \equiv 2\left(\bmod p^{2}\right)$ and

$$
p \geqq 2 r^{2}+1 .
$$

7. As remarked in the Introduction, if $m$ in (1.4) or (1.5) is restricted to an arithmetic progression, the theorems of this paper may not hold. We shall illustrate in a special case. It is clear, to begin with, that if 


$$
e_{m_{0}+p-1} \equiv e_{m_{0}}\left(\bmod p^{2}\right),
$$

where $m_{0}$ is a fixed number $\geqq 2$, then it follows by repeated application of the congruence

$$
e_{m+2 p-2}-2 e_{m+p-1}+e_{m} \equiv 0\left(\bmod p^{2}\right) \quad(m \geqq 2)
$$

that

$$
e_{m+p-1} \equiv e_{m}\left(\bmod p^{2}\right)
$$

for $m \equiv m_{0}(\bmod p-1), m \geqq m_{0}$.

In particular, since

$$
E_{p+1} \equiv E_{2} \equiv-1(\bmod p)
$$

we can determine an integer $c$ such that

$$
c^{p-1} E_{p+1} \equiv-1\left(\bmod p^{2}\right) .
$$

Hence, if we put

$$
e_{m}=c^{m} E_{m},
$$

we have

$$
e_{p+1} \equiv e_{2}\left(\bmod p^{2}\right)
$$

from which it follows that

$$
e_{m+p-1} \equiv e_{m}\left(\bmod p^{2}\right)
$$

for all

$$
m \equiv 2(\bmod p-1)
$$$$
m \geqq 2 \text {. }
$$

This proves

TheOREM 9. The numbers $e_{m}$ defined by (7.1) and (7.2) satisfy (7.3) for all $m$ in the arithmetic progression (7.4).

\section{REFERENCES}

1. A. Brauer, Elementary estimates for the least primitive root, Studies in Mathematics and Mechanics presented to Richard von Mises, New York, 1954, pp. 20-29.

2. N. Nielsen, Traité élémentaire des nombres de Bernoulli, Paris, 1923.

3. N. E. Nörlund, Vorlesungen über Differenzenrechnung, Berlin, 1924.

4. H. S. Vandiver, An aspect of the linear congruence with applications to the theory of Fermat's quotient, Bull. Amer. Math. Soc. vol. 22 (1916) pp. 61-67.

DUke UNIVERSITY, Durham, North Carolina

State. College of Agriculture and Engineering, Raleigh,'North Carolina 